OPEN ACCESS

Edited by:

Jeff Guo,

University of Cincinnati, United States

Reviewed by:

George Bertsias,

University of Crete, Greece

Vladimir Tesar,

Charles University, Czechia

*Correspondence:

Eric HY Lau

ehylau@hku.hk

Zhiming Lin

Izm-zj99@163.com

${ }^{+}$These authors have contributed equally to this work

Specialty section:

This article was submitted to

Drugs Outcomes Research and

Policies,

a section of the journal

Frontiers in Pharmacology

Received: 09 March 2021

Accepted: 20 August 2021

Published: 06 September 2021

Citation:

Dai Z, Zhang X, Wong IOL, Lau EHY and Lin $Z$ (2021) Treatment for Severe Lupus Nephritis: A Cost-Effectiveness Analysis in China.

Front. Pharmacol. 12:678301. doi: 10.3389/fphar.2021.678301

\section{Treatment for Severe Lupus Nephritis: A Cost-Effectiveness Analysis in China}

\author{
Zonglin $\mathrm{Dai}^{1+}{ }^{1+}$ Xi Zhang ${ }^{2 \dagger}$, Irene OL Wong ${ }^{1}$, Eric HY Lau ${ }^{1,3 *}$ and Zhiming Lin ${ }^{2 *}$ \\ ${ }^{1}$ School of Public Health, Li Ka Shing Faculty of Medicine, The University of Hong Kong, Pokfulam, Hong Kong, SAR China, \\ ${ }^{2}$ Division of Rheumatology, Third Affiliated Hospital of Sun Yat-sen University, Guangzhou, China, ${ }^{3}$ Laboratory of Data Discovery \\ for Health (D24H), Hong Kong Science and Technology Park, Hong Kong, Hong Kong, SAR China
}

Background: Lupus nephritis $(\mathrm{LN})$ is the most common secondary glomerular diseases that will cause end-stage renal disease (ESRD) and renal-related death. The costeffectiveness of various treatments for $L N$ recommended by official guidelines has not been investigated in China. Our study is to evaluate clinical prognosis and costeffectiveness of the current treatments for severe LN.

Methods: A Markov model was simulated for 1,000 LN patients of 30 years old, over a 3years and 30-years lifetime horizon respectively. We assessed the cost-effectiveness of six therapeutic strategies from a societal perspective, with cyclophosphamide (CYC) or mycophenolate mofetil (MMF) induction therapy followed by CYC, MMF or azathioprine (AZA) maintenance therapy. Main outcomes included quality-adjusted life years (QALYs), incremental cost-effectiveness ratio (ICER) and clinical prognosis. One and three times gross domestic product (GDP) per capita were used as the willingness-to-pay (WTP) thresholds. We also carried out sensitivity analysis under a lifetime horizon.

Results: Compared with the baseline strategy of CYC induction and maintenance, for a 3years horizon the most cost-effective strategy was CYC induction and AZA maintenance with $\$ 448$ per QALY gained, followed by MMF induction and AZA maintenance which however was not cost-effective under the one times GDP per capita WTP threshold. For a lifetime horizon, CYC induction and AZA maintenance remained the most cost-effective strategy but MMF induction and maintenance became cost-effective under the one times GDP per capita WTP threshold and achieved a higher complete remission rate $(57.2$ versus $48.9 \%$ ) and lower risks of ESRD (3.3 versus 5.8\%) and all-cause mortality (36.0 versus $40.8 \%)$. The risk of developing ESRD during maintenance was the most influential parameter affecting ICER.

Conclusions: The strategy of CYC induction followed by AZA maintenance was the most cost-effective strategy in China for short-term treatment, while the strategy of MMF in both induction and maintenance became cost-effective and yielded more desirable clinical outcomes for lifetime treatment. The uncertainty analysis supported the need for monitoring the progression to ESRD.

Keywords: Cost-Effectiveness, severe lupus nephritis, Markov model, therapeutic strategies, clinical prognosis 


\section{INTRODUCTION}

Lupus nephritis (LN) is a common complication of systemic lupus erythematosus (SLE), a chronic inflammatory disease that may induce organ damage, typically the kidney. The frequency of developing LN from SLE varies worldwide, with $40-80 \%$ among Asians (Almaani et al., 2017). In China, LN has become the most common secondary glomerular diseases, accounting for over $50 \%$ of adults with SLE (Chinese Guidelines for Diagnostic and Treatment of Lupus Nephritis Writing Group, 2019). The standardized mortality ratio of LN patients was around six compared with the general population (Yap et al., 2012; Parikh et al., 2020). 10\% of LN patients developed end-stage renal disease (ESRD) and mortality due to kidney disease was found to be $5-25 \%$ for patients with proliferative LN (Almaani et al., 2017; Parikh et al., 2020).

According to the latest treatment guidelines for $\mathrm{LN}$ from Chinese Medical Association (2019) (CMA) (Chinese Guidelines for Diagnostic and Treatment of Lupus Nephritis Writing Group, 2019), LN is classified into class I (minimal mesangial LN) to class VI (advanced sclerosing LN). The currently recommended first-line treatments include the basic treatment, hydroxychloroquine (HCQ) and glucocorticoids (GC), plus immunosuppressive (IS) therapy which mainly consists of cyclophosphamide (CYC), azathioprine (AZA) and mycophenolate mofetil (MMF). The American College of Rheumatology (2012) (ACR), the European League Against Rheumatism (2019) (EULAR) and GLADEL-PANLAR Latin American (2018) provided similar recommendations (Hahn et al., 2012; Pons-Estel et al., 2018; Fanouriakis et al., 2019). In general, patients diagnosed with class III (focal LN with less than $50 \%$ of glomeruli), class IV (diffuse LN with over 50\% glomeruli) and class V LN (subepithelial immune deposits and membranous LN) in combination with class III or IV require more aggressive therapy, i.e. using IS drugs in additional to the basic treatment. Besides, class III and class IV patients account for 39-72\% of all six pathologic types (Wang et al., 2018). Therefore, we focused on class III and IV LN patients, including class III/IV + V (hereafter referred as 'severe LN patients').

A two-phase paradigm was recommended for severe LN patients. In the first phase, patients received induction therapy to control the acute inflammatory injury of the kidney and to achieve complete remission (defined as urine protein-tocreatinine ratio $<0.5 \mathrm{mg} / \mathrm{mmol}$ with normal kidney function). The second phase is maintenance therapy, targeting at keeping complete remission and avoiding recurrence. It is common that LN patients received lifelong treatment which led to profound economic burden. In the United States (US), the annual medical expenditures of LN exceeded \$46,000 (USD) per patient (Carls et al., 2009). Another report estimated that the total annual costs including outpatient, hospitalization, non-medical costs and indirect costs of SLE was over $\$ 6,000$ (USD) in Shanghai, China (Zhang et al., 2017).

CYC and MMF are listed as the first-line drugs in induction therapy, and AZA and MMF are recommended in the maintenance phase. Although CYC was not recommended for the maintenance therapy by the guidelines, it is still used in China due its relatively low cost (Zhang et al., 2014).

To our knowledge, no integrated cost-effectiveness analysis has been carried out considering the two phases of induction and maintenance and their interplay. We designed this study with structured model and surveillance of ESRD and death to assess the cost-effectiveness of current LN treatment strategies in China.

\section{MATERIALS AND METHODS}

\section{Target Population and Therapeutic Strategies}

Patients primarily diagnosed with class III, IV LN alone, and in combination with class $\mathrm{V}$ were targeted in our study. Milder class I and class II (mesangial proliferative LN) patients require basic treatment of HCQ and GC only without IS therapy. More severe class $\mathrm{V}$ (membranous LN) patients were treated based on their patient conditions and class VI (advanced sclerosing LN) patients require renal replacement therapy instead of using IS drugs. These patients were not considered in our study.

We referred to the CMA treatment guidelines for severe $\mathrm{LN}$ patients and current clinical practice in China in our study (Hahn et al., 2012; Pons-Estel et al., 2018; Chinese Guidelines for Diagnostic and Treatment of Lupus Nephritis Writing Group, 2019; Fanouriakis et al., 2019). We considered intravenous CYC which is the main route of administration for treating LN patients in China (Chinese Guidelines for Diagnostic and Treatment of Lupus Nephritis Writing Group, 2019). The recommended dosage is $0.5-1 \mathrm{~g}$ per month for CYC and $1.5-3 \mathrm{~g} /$ day for MMF as the first-line IS drugs to treat LN during the 6months induction therapy. Besides, patients treated with CYC or MMF also received HCQ (0.3-0.5 g/ day) and pulse GC $(0.5-1 \mathrm{~g} /$ day $)$ for 3 days, followed by prednisone $(0.5-1 \mathrm{mg} /$ $\mathrm{kg} /$ day), reducing the dose gradually each month until $0.15 \mathrm{mg} / \mathrm{kg} /$ day. In the maintenance therapy, AZA and MMF were recommended as the first-line IS drugs with dosage $75-100 \mathrm{mg} /$ day and less than $2 \mathrm{~g} /$ day respectively. The dosage of CYC in maintenance was $0.5-1.0 \mathrm{~g} / \mathrm{m}^{2}$ every 3 months (Contreras et al., 2004).

We assumed that the standard treatment (HCQ and GC) was used during the entire treatment. CYC or MMF was used for 6 months in initial induction therapy. If complete remission is achieved, patients will switch to maintenance therapy with CYC, AZA or MMF (Fanouriakis et al., 2019). Patients experiencing renal relapse after complete remission during the maintenance therapy were assumed to switch back to the same initial regimen as in the induction therapy. However, if complete remission is not achieved or only partial remission (defined as $\geq 50 \%$ reduction in proteinuria to subnephrotic levels) is achieved by one of the IS drugs (CYC or MMF) after 6 months, the same induction therapy is then extended for another 6 months. However, a switch to the other IS drug will be implemented if one-year induction fails under the same therapy (Chinese Guidelines for Diagnostic and Treatment of Lupus Nephritis Writing Group, 2019). According to ACR treatment guideline, rituximab (RTX) was typically used 


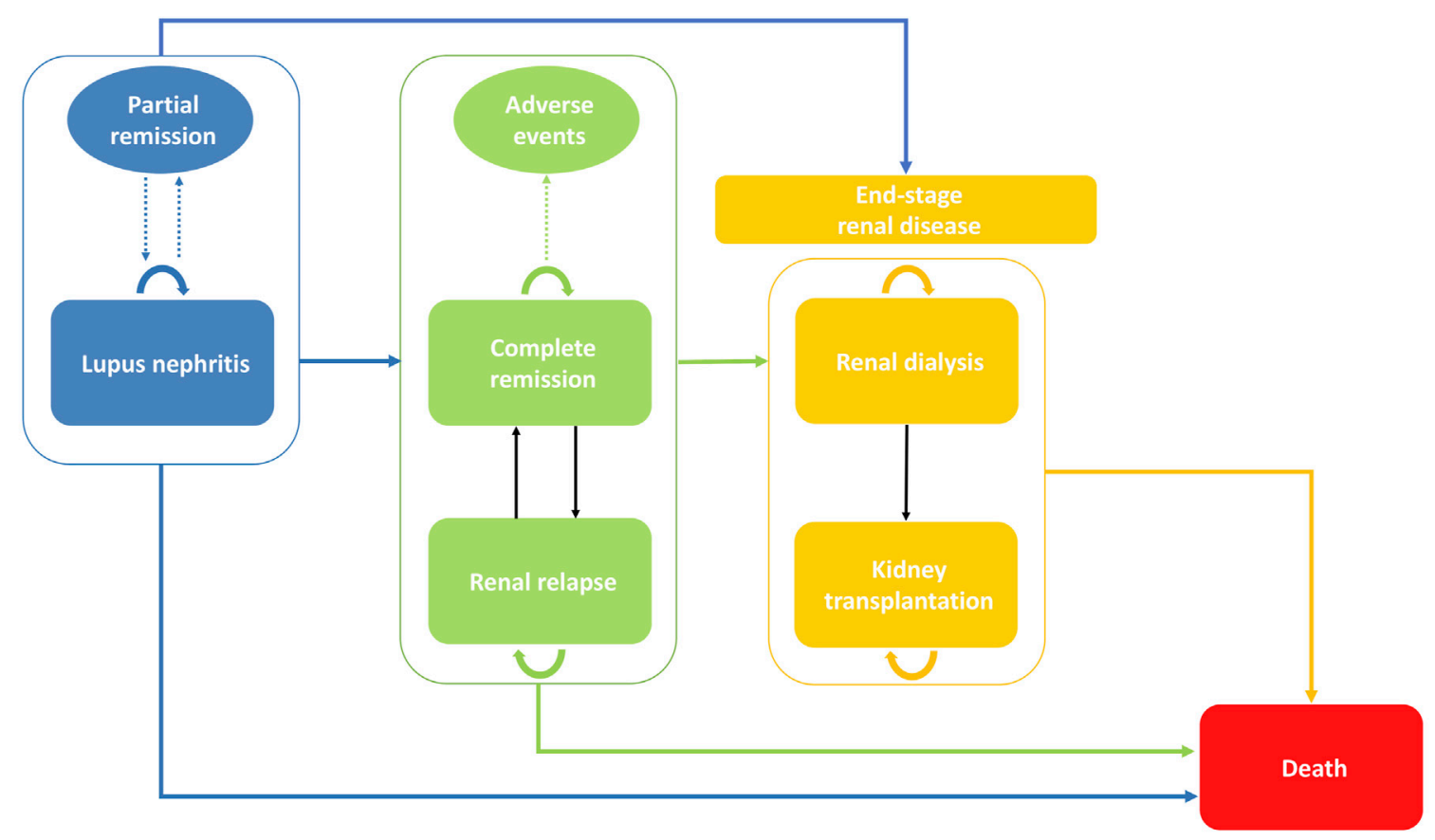

FIGURE 1 | Markov structural model of health states with disease progression with 6-months cycles. The rounded rectangles represent health states and the ovals represent the potential outcomes in each phase. Transitions between phases or between states were indicated by solid arrows. The dotted arrows indicated potential outcomes. The initial phase is induction phase where patients start to receive therapy. After achieving complete remission, patients will progress to the maintenance therapy. Patients in both induction and maintenance phase may transit to renal replacement phase. All health states have a risk of death.

when both CYC and MMF fail, and we assumed that patients could not achieve complete remission if RTX also fail (Hahn et al., 2012).

Severe LN patients should always be treated with IS drugs in additional to standard treatment (Hahn et al., 2012). CYC has long been considered the gold standard in treating LN, with superior complete remission rate and cheapest direct treatment cost, hence it is still widely used in China to achieve renal remission and prevent renal flares, although it is associated with adverse events (AEs) including bone marrow suppression, infertility and malignancy. Hence, we defined baseline strategy (S1) as: initial induction with CYC followed by CYC maintenance (CYC $\rightarrow$ CYC). We considered other strategies which comprised of combinations between two drug choices (CYC and MMF) in the initial induction and three drug choices (CYC, AZA and $\mathrm{MMF}$ ) in the maintenance phase (S2-S6) for comparison, namely $\mathrm{MMF} \rightarrow \mathrm{CYC}, \quad \mathrm{CYC} \rightarrow \mathrm{AZA}, \quad \mathrm{MMF} \rightarrow \mathrm{AZA}, \quad \mathrm{CYC} \rightarrow \mathrm{MMF}$ and $\mathrm{MMF} \rightarrow \mathrm{MMF}$.

\section{The Analytic Model Model Overview}

A Markov model was designed to assess the cost-effectiveness of six therapeutic strategies for LN. The mean age of patients diagnosed with LN was around 30 years (Nossent and Koldingsnes, 2000; Dooley et al., 2011; Moroni et al., 2014; Wang et al., 2018), accordingly we simulated patients who met the treatment standards of severe LN from the same age. A lifetime horizon was modeled, given that continuous immunosuppressive therapy is needed to reduce SLE activity and ESRD and improve the quality of life for severe LN patients (Maroz and Segal, 2013). The life expectancy of Chinese LN patients was around 60 years, hence the lifetime horizon was set to be 30 years (Mok et al., 2013). The specific timeline of treatment has not been clearly stipulated by guidelines but it is recommended to receive at least 3-years maintenance. Therefore, we also evaluated cost-effectiveness over 3 years to assess the short-term outcomes. We adopted a societal perspective in the study and considered both direct and indirect costs. The transition period or cycle of the model was 6-months covering the induction period and evaluation of the therapy (Dooley et al., 2011). Hence in the model, we ran a total of 60 cycles to simulate the lifetime effect of disease progression with different treatment strategies. Main outcomes from the model included the cost of each patient, cumulative quality-adjusted life years (QALYs), incremental cost per QALY and incremental cost-effectiveness ratio (ICER). ICER indicated additional costs per QALY gained compared with the previous least costly strategy. We also simulated the disease trends of ESRD and death.

\section{Model Structure}

We considered four main phases of patient management, namely induction, maintenance, renal replacement, and terminal phase, 
TABLE 1 | Transition probabilities related to disease progress and different treatments ${ }^{a}$.

\begin{tabular}{|c|c|c|c|}
\hline 6-months transition & Estimate (\%) & $\begin{array}{c}\text { Range for sensitivity } \\
\text { analysis }^{\mathbf{b}}(\%)\end{array}$ & References \\
\hline \multicolumn{4}{|c|}{ Induction therapy with immunosuppressive drugs, from lupus nephritis } \\
\hline To complete remission with CYC & 40.84 & $21.74-66.67$ & Moroni et al. (2014) \\
\hline To complete remission with MMF & 31.37 & $25.00-54.00$ & Moroni et al. (2014) \\
\hline To complete remission with RTX & 45.78 & $14.20-72.70$ & Moroni et al. (2014) \\
\hline To ESRD & 0.80 & $0.71-0.84$ & Croca et al. (2011) \\
\hline To lupus-related death & 0.80 & $0.48-1.87$ & Croca et al. (2011) \\
\hline To ESRD, when treatment failure ${ }^{c}$ & 2.48 & $0.81-8.00$ & Korbet et al. (2000) \\
\hline To lupus-related death, when treatment failure & 2.83 & $2.14-3.64$ & Korbet et al. (2000) \\
\hline \multicolumn{4}{|c|}{ Maintenance therapy with immunosuppressive drugs, from complete remission } \\
\hline To renal relapse with CYC & 5.00 & $3.30-7.73$ & Nee et al. (2015); Pons-Estel et al. (2018) \\
\hline To ESRD with CYC & 0.45 & $0.23-0.96$ & Zhang et al. (2014) \\
\hline To lupus-related death with CYC & 1.84 & $0.33-13.08$ & Nee et al. (2015); Tunnicliffe et al. (2018) \\
\hline To renal relapse with AZA & 3.64 & $2.34-5.87$ & Nee et al. (2015) \\
\hline To ESRD with AZA & 0.30 & $0.06-1.60$ & Nee et al. (2015) \\
\hline To lupus-related death with AZA & 0.25 & $0.04-1.57$ & Nee et al. (2015) \\
\hline To renal relapse with MMF & 1.85 & $1.22-2.86$ & Nee et al. (2015) \\
\hline To ESRD with MMF & 0.12 & $0.02-0.63$ & Nee et al. (2015) \\
\hline To lupus-related death with MMF & 0.43 & $0.07-2.85$ & Nee et al. (2015) \\
\hline \multicolumn{4}{|l|}{ Renal replacement therapy } \\
\hline Transition probability to KT after receiving renal dialysis & 0.85 & $0.36-2.01$ & Yikui et al. (2015); Wang et al. (2019) \\
\hline Transition probability to death after receiving renal dialysis & 4.29 & $1.00-7.47$ & Wu et al. (2014); Tsai et al. (2019) \\
\hline Transition probability to death after receiving $\mathrm{KT}$ & 0.29 & $0.19-0.37$ & Wu et al. (2014); Tsai et al. (2019) \\
\hline
\end{tabular}

with six health status including LN, complete remission, renal relapse, renal dialysis, kidney transplantation and death in our model (Figure 1). All severe LN patients received the induction therapy. Patients who achieved complete remission would progress to the maintenance phase but may still have a risk of relapse. A systematic review and meta-analysis found that renal dialysis was always considered as the initial renal replacement therapy, prior to transplantation (Swai et al., 2020). For simplicity, we assumed in our model that patients with ESRD received dialysis first, and may further require kidney transplantation if the patient's condition deteriorated (Adler et al., 2006).

\section{Model Input}

\section{Transition Probability}

We extracted the relevant transition probabilities between health status and their ranges for uncertainty analysis, based on an extensive literature review of primary studies and meta-analyses (Table 1). We extracted the drug efficiency data in induction phase, prioritizing head-to-head comparison studies. These parameters were converted for use in our model with a 6-months cycle (detailed description in the Supporting Material).

According to the guideline in from CMA, after the induction therapy, standard evaluation methods including clinical symptoms (kidney function) and indicators (urine protein-tocreatinine ratio) are used to measure the health status. Those who enter the maintenance therapy after reaching complete remission must have matched the above assessment (Chinese Guidelines for Diagnostic and Treatment of Lupus Nephritis Writing Group, 2019). In the simulation, we assumed that the effect of maintenance therapy was independent of previous states during induction (Chinese Guidelines for Diagnostic and Treatment of Lupus Nephritis Writing Group, 2019).

We searched PubMed, Web of Science, Google Scholar, China National Knowledge Infrastructure and Embase for articles with the keywords "lupus nephritis", "induction", "cyclophosphamide", "mycophenolate mofetil" or/and "rituximab" in induction therapy. A similar search was carried out by changing the keyword "induction" to "maintenance", and added "azathioprine" in maintenance therapy. In renal replacement phase, we used the keywords "end-stage renal disease", "kidney transplantation", "death" or/and "mortality" without restriction on language between 1980 and 2020. To obtain head-to-head transition probability, we reviewed the literature extensively. For instance, in induction therapy, transitions from LN to complete remission including CYC, MMF and RTX therapy were based on a clinical observational study, with converting the annual transition probability to 6months cycle (Moroni et al., 2014).

\section{Quality-Adjusted Life Years and Costs}

We used QALYs as the utility measurement, calculated by multiplying the utility score by time spent in a state (Whitehead and Ali, 2010). The utility scores of various health status measured by EQ-5D index were extracted from the 
literature (Liem et al., 2008; Mohara et al., 2014). The state of death was assigned a utility score of 0 , and the other states were assigned health utility score ranging from 0.56 to 0.94 (Supplementary Table S1). Due to the higher treatment costs for AEs and the significant infertility risk due to CYC, the estimated utility score for complete remission and renal relapse after being treated with CYC was lower than that treated with other IS drugs (McDermott and Powell, 1996; Nee et al., 2015; Jones et al., 2019).

In the model, we considered total costs associated with treatment and management of LN, including direct costs and indirect costs. Direct costs consisted of direct health care costs (drugs, treatment-related AEs, medical devices, diagnostic tests, laboratory tests, hospital admission fee, etc.) and direct nonmedical costs (transportation, accommodation expenses and social service such as retraining). AEs included major and minor infections, pneumonia, gastrointestinal manifestation, and leucopenia induced by IS drugs, and diabetes, hypertension, fractures, and eye diseases induced by GC and HCQ. Risks of these AEs and the related costs were presented in (Supplementary Tables S2-4). Prices of GC and HCQ and IS drugs were obtained from Hospital Information System, the Third Affiliated Hospital of Sun Yat-sen University, a major medical center in China and the evaluation of the costs of AE was also based on the system by two physicians (XZ and ZL) (The Third Affiliated Hospital of Sun Yat-sen University, 2019). Indirect costs included productivity loss, calculated by multiplying gross value of daily average income per capita in China by days off work (Supplementary Table S5); (Jo, 2014). The major cost for living donor kidney transplantation was accrued shortly after the treatment, and the direct and indirect health costs dropped quickly afterwards (Supplementary Table 1).

Considering the similar utilities between hemodialysis (HD) and peritoneal dialysis (PD) and the more popular use of HD in China (Wang et al., 2006; Liem et al., 2008; Zhang and Zuo, 2016). we considered the costs of HD for patients with ESRD in the analysis. The costs of dialysis in renal replacement phase included medication, consultation, laboratory and radiological investigation, dialysis solution, machine depreciation and other costs. Similarly, we considered the costs of living donor kidney transplantation in the study which is most common in China. Costs of kidney transplantation also included surgical and nursing, laboratory and testing, immunosuppressive agents, accommodation and other costs (Xiaoming et al., 2012). All costs were converted to 2019 prices using the consumer price indices from 2003 to 2019, and from Chinese Yuan (CNY, $¥)$ to U.S. dollars (USD, \$) using the exchange rate in 2019 (1 USD = 6.87 CNY) (National Bureau of Statistics of China, 2019). QALY and costs were discounted at a rate of $1.5 \%$ per 6-months cycle (3\% per year) (Chhatwal et al., 2016). One times gross domestic product (GDP) per capita ( $¥ 70,892$ or US\$10,319, 2019) in mainland China was considered as the willingness-to-pay (WTP) threshold, which was considered highly cost-effective, and three times GDP per capita was also adopted as the threshold for being cost-effective ( $¥ 212,676$ or US $\$ 30,957,2019$ ) (Marseille et al., 2015; National Bureau of Statistics of China, 2019).

\section{Uncertainty Analysis}

We assessed the uncertainty of the estimates with deterministic sensitivity analysis (DSA) and probabilistic sensitivity analysis (PSA) over the lifetime horizon. Key parameters including the transition probability between health states, costs and utility of each health state and discount rate were varied sequentially in DSA (Table 1; Supplementary Table S1). Ranges for the uncertainty analysis were either obtained from the range of estimates in systematic reviews, or from the 95\% confidence intervals from a specific study. The outcome was presented in tornado plots, showing the most influential parameters on model results. In PSA, pre-defined parameters were re-sampled from respective distributions with 1,000 simulated cohorts. Dirichlet, binomial, normal and gamma distributions were assumed in the transition probabilities between states, discount rate, utility and costs respectively (Chen et al., 2016).

\section{RESULTS}

Table 2 summarized cost effectiveness and outcomes of different LN treatment strategies by 3 -years and lifetime horizon. The efficiency frontiers at lifetime horizons are presented in Supplementary Figure S1. For a 3-years horizon, treating LN patients with CYC induction therapy and AZA maintenance therapy (S3, \$448 per QALY gained) was most cost-effective compared with the baseline strategy $(\mathrm{S} 1: \mathrm{CYC} \rightarrow \mathrm{CYC})$, with 0.218 more QALYs (Table 2). S4 (MMF $\rightarrow$ AZA) was the next costeffective strategy if one is willing to pay $\$ 22,262$ more than $S 3$ $(\mathrm{CYC} \rightarrow \mathrm{AZA})$ at a WTP of three times GDP per capita (US\$30,957) but was then dominated by the most effective strategy S6 (MMF $\rightarrow$ MMF) after 24 years (Supplementary Figure S2). The proportions of patients experiencing renal replacement was lower for strategies with MMF maintenance (S5 and S6), and there were lower complete remission rate and higher all-cause mortality for strategies with CYC maintenance (S1 and S2, Table 2). For a lifetime horizon, CYC induction and AZA maintenance therapy (S3) was also the most cost-effective strategy which was associated with 48.9, 5.8 and $40.8 \%$ complete remission rate, risk of renal replacement and all-cause mortality respectively. However, S6 (MMF $\rightarrow \mathrm{MMF})$ achieved the highest complete remission rate and the lowest risk of renal replacement and all-cause mortality, at 57.2, 3.3 and $36.0 \%$ respectively among all cost-effective strategies. Again, strategies with CYC maintenance (S1 and S2) had noticeably lower complete remission rate and higher mortality. Due to the small number of cases who developed ESRD (Supplementary Figure S3, costs were mainly driven drug costs (CYC, MMF or AZA). Cost associated with ESRD increased disproportionately in the long run but still much lower than the drug costs.

\section{Sensitivity Analysis}

We conducted DSA for the most cost-effective strategy S3 $(\mathrm{CYC} \rightarrow \mathrm{AZA})$ for a lifetime horizon (Figure 2). The most influential parameter that affected ICER was the risk of ESRD after complete remission during AZA maintenance therapy. Other influential parameters included the mortality risk 
TABLE 2 | Base-case cost-effectiveness outcomes of different strategies for $L N$ treatment, and predicted cumulative incidence of complete remission, renal replacement and all-cause mortality.

\begin{tabular}{|c|c|c|c|c|c|c|c|c|}
\hline Strategy & $\begin{array}{c}\text { Cumulative } \\
\text { costs } \\
\text { (US\$) }\end{array}$ & $\begin{array}{c}\text { Cumulative } \\
\text { QALYs }\end{array}$ & $\begin{array}{l}\text { Incremental } \\
\text { costs } \\
\text { (US\$) }\end{array}$ & $\begin{array}{c}\text { Incremental } \\
\text { QALYs }\end{array}$ & $\begin{array}{l}\text { ICER } \\
\text { (US\$/ } \\
\text { QALY) }\end{array}$ & $\begin{array}{c}\text { Complete } \\
\text { remission }^{\mathrm{a}} \\
(\%)\end{array}$ & $\begin{array}{c}\text { Renal } \\
\text { replacement }^{\text {b }} \\
(\%)\end{array}$ & $\begin{array}{c}\text { All-cause } \\
\text { mortality }^{c} \\
(\%)\end{array}$ \\
\hline \multicolumn{9}{|l|}{ 3-years horizon } \\
\hline S1: CYC $\rightarrow$ CYC & 15,874 & 2.156 & - & - & - & 75.9 & 2.9 & 7.9 \\
\hline S3: $C Y C \rightarrow A Z A$ & 15,972 & 2.374 & 98 & 0.218 & 448 & 82.5 & 2.5 & 3.4 \\
\hline $\begin{array}{l}\text { S2: } \\
\mathrm{MMF} \rightarrow \mathrm{CYC}\end{array}$ & 17,469 & 2.308 & 1,595 & 0.152 & Dominated & 75.4 & 3.0 & 7.6 \\
\hline S4: MMF $\rightarrow$ AZA & 17,484 & 2.442 & 1,512 & 0.068 & 22,262 & 81.8 & 2.6 & 3.5 \\
\hline $\begin{array}{l}\text { S5: } \\
\text { CYC } \rightarrow \mathrm{MMF}\end{array}$ & 17,594 & 2.384 & 1,622 & 0.010 & Dominated & 85.9 & 2.0 & 3.2 \\
\hline $\begin{array}{l}\text { S6: } \\
\text { MMF } \rightarrow \text { MMF } \\
\text { Lifetime horizon }\end{array}$ & 18,897 & 2.452 & 1,413 & 0.010 & 136,075 & 85.3 & 2.1 & 3.4 \\
\hline $\begin{array}{l}\text { S1: } \\
\mathrm{CYC} \rightarrow \mathrm{CYC}\end{array}$ & 70,286 & 9.745 & - & - & - & 18.7 & 5.1 & 73.7 \\
\hline $\begin{array}{l}\text { S2: } \\
\mathrm{MMF} \rightarrow \mathrm{CYC}\end{array}$ & 76,480 & 10.702 & 6,194 & 0.957 & Dominated & 18.3 & 5.2 & 73.2 \\
\hline $\begin{array}{l}\text { S3: } \\
\text { CYC } \rightarrow \text { AZA }\end{array}$ & 82,540 & 14.287 & 12,254 & 4.542 & 2,698 & 48.9 & 5.8 & 40.8 \\
\hline $\begin{array}{l}\text { S4: } \\
\text { MMF } \rightarrow \text { AZA }\end{array}$ & 88,393 & 14.866 & 5,853 & 0.579 & Dominated & 47.1 & 6.0 & 41.4 \\
\hline $\begin{array}{l}\text { S5: } \\
\text { CYC } \rightarrow \text { MMF }\end{array}$ & 90,031 & 14.869 & 7,491 & 0.582 & Dominated & 58.5 & 3.2 & 35.5 \\
\hline $\begin{array}{l}\text { S6: } \\
\mathrm{MMF} \rightarrow \mathrm{MMF}\end{array}$ & 93,708 & 15.517 & 11,168 & 1.230 & 9,079 & 57.2 & 3.3 & 36.0 \\
\hline
\end{tabular}

AZA, azathioprine; CYC, cyclophosphamide; ICER, incremental cost-effectiveness ratios; MMF, mycophenolate mofetil; QALYS, quality-adjusted life years.

${ }^{a}$ Chi-squared test for equality of proportions: $\mathrm{p}<0.001$ among strategies at 3-years horizon and $\mathrm{p}<0.001$ among strategies at lifetime horizon.

${ }^{b}$ Renal replacement included renal dialysis and kidney transplantation. Chi-squared test for equality of proportions: $\mathrm{p}=0.658$ among strategies at $3-y e a r s ~ h o r i z o n$ and $\mathrm{p}=0.005$ among strategies at lifetime horizon.

${ }^{c}$ Chi-squared test: $\mathrm{p}<0.001$ among strategies at 3-years horizon and $\mathrm{p}<0.001$ among strategies at lifetime horizon.

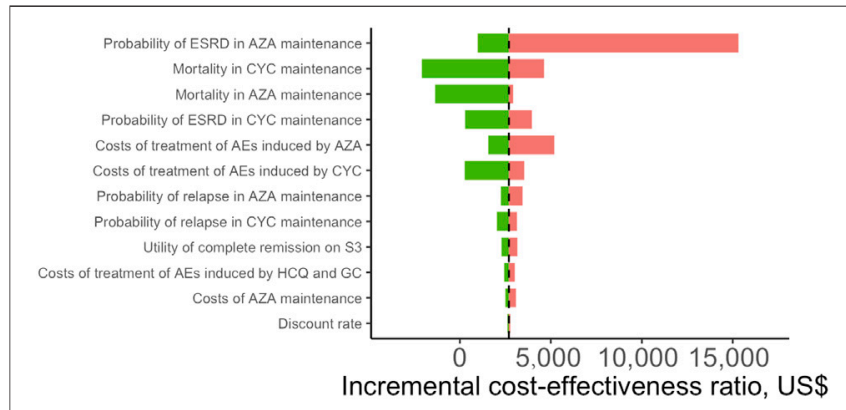

FIGURE 2 | Tornado plot of deterministic sensitivity analysis for patients with lupus nephritis receiving the most cost-effective strategy (S3: CYC $\rightarrow$ AZA) compared with the baseline strategy $(\mathrm{S} 1: \mathrm{CYC} \rightarrow \mathrm{CYC})$ over a lifetime horizon. The base-case result is presented by vertical dashed line. The length of the bars reflects the degree of parameters that influence quality-adjusted life years. Only the top 12 most influential parameters were presented. HCQ, hydroxychloroquine; GC, glucocorticoids; CYC, cyclophosphamide; AZA, azathioprine; ESRD, end-stage renal disease; AEs, adverse events.

associated with CYC and AZA maintenance therapy, probability of ESRD in CYC maintenance therapy, and costs of treatmentrelated AEs by AZA and CYC. In PSA we estimated that at the WTP of one times GDP per capita, most simulated cohorts treated with S6 (MMF in both treatment phases) over a lifetime horizon were under the ceiling ratio, and more than
$99 \%$ of the cohorts were under the ceiling ratio for the three times GDP per capita WTP, meaning the cost-effectiveness. S6 had the highest acceptability of $34 \%$ among all strategies, followed by S4 $(\mathrm{MMF} \rightarrow \mathrm{AZA})$ being cost-effective with $28 \%$ probability (Figure 3). The cost-effectiveness acceptability of S4 $(\mathrm{MMF} \rightarrow \mathrm{AZA})$ and $\mathrm{S} 5(\mathrm{CYC} \rightarrow \mathrm{MMF})$ became stable whereas the probability of being cost-effective for S6 increased to $40 \%$ at the three times GDP per capita WTP threshold.

\section{DISCUSSION}

LN with subsequent development of ESRD has led to substantial mortality burden among patients with SLE (Almaani et al., 2017). Current LN therapies may cause complications such as infections, pneumonia, toxic retinopathy and diabetes which require further treatment and are associated with high financial burden. While various $\mathrm{LN}$ treatment options have been recommended, our study is first to evaluate the cost-effectiveness of these treatment strategies in an integrated framework considering induction, maintenance, renal replacement and terminal phases in China.

We found that the strategy of CYC induction followed by AZA maintenance therapy (S3) was the most cost-effective for both the 3 -years and lifetime horizon. A study in Thailand found that the same strategy was the only cost-saving strategy (Mohara et al., 2014). The most effective strategy S6 (MMF $\rightarrow$ MMF) was not 


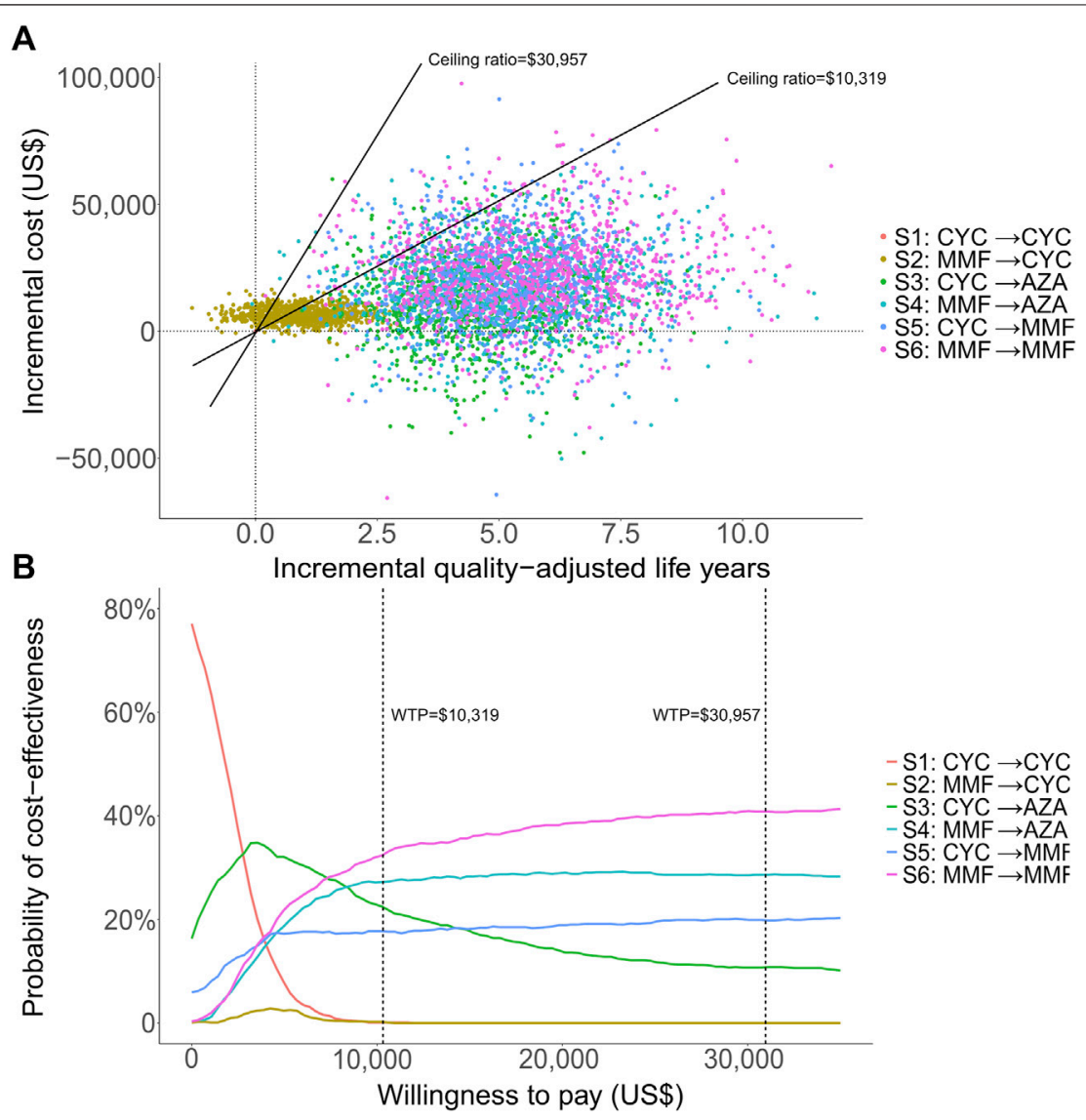

FIGURE 3 | Probabilistic analytic results for the scenario over a lifetime horizon: the incremental cost-effectiveness differences simulated with 1,000 patients (A) and the cost-effectiveness acceptability curves of all strategies (B). One-time and three-time gross domestic product per capita were used for the willingness to pay thresholds, at US\$10,319 and US\$30,957 respectively.

cost-effective at both WTP thresholds for a 3-years time horizon. However, it became affordable and most cost-effective for a lifetime horizon probably due to a lower relapse rate and risk of developing ESRD for MMF maintenance compared to AZA, which compensated the relatively high drug cost for MMF (Dooley et al., 2011). The cost-effective treatment identified in our study is likely applicable in the Asian settings. Although no similar costeffectiveness study has been conducted in high income countries considering the induction and maintenance therapy together, several studies examined cost-effectiveness of the induction therapy and maintenance therapy separately. In the United Kingdom, MMF is costing US $\$ 3,100$ less than CYC over the 24-week period in induction therapy based on the price in 2005 (Wilson et al., 2007). In the United States, MMF was found to be more cost-effective, with an ICER of \$6,454/QALY compared to AZA in lifetime maintenance therapy (Nee et al., 2015). These studies showed that MMF was cost-effective for both induction and maintenance therapy, consistent with our results. Our study results are likely applicable to high and middle income countries.

Compared with other strategies, MMF maintenance was associated with the lowest risks of ESRD and death over 30 years (Table 2; Supplementary Figure S3). S5 $(\mathrm{CYC} \rightarrow \mathrm{MMF})$ was also dominated by $\mathrm{S} 6(\mathrm{MMF} \rightarrow \mathrm{MMF})$ in our study. Further, $\mathrm{S} 3(\mathrm{CYC} \rightarrow \mathrm{AZA})$ and $\mathrm{S} 4(\mathrm{MMF} \rightarrow \mathrm{AZA})$ resulted in higher risk of ESRD than $\mathrm{S} 6(\mathrm{MMF} \rightarrow \mathrm{MMF})$ when longer course of treatment was adopted, with the discrepancy becoming more prominent starting from 5 years of treatment (Supplementary Figure S3). This was partly due to the higher renal relapse rate in AZA maintenance, which was also demonstrated by a previous systematic review (Tunnicliffe et al., 2018). We also showed that use of CYC in long-term maintenance therapy would result in lower complete remission rate, and higher risk of ESRD and death. A meta-analysis also found that using MMF was likely to produce better clinical outcome than CYC (Liu et al., 2012). In China, AZA treatment is subsidized, and the use of CYC maintenance for treating sever LN patients should be discouraged.

Disease progression rate to ESRD during AZA maintenance was found to be the most influential factor affecting the cost-effectiveness of S3 (CYC $\rightarrow$ AZA) (Figure 2). Clinically, identifying patients with higher risk of developing ESRD is important to reduce the risk of morbidity and mortality, which was also an important factor affecting cost-effectiveness. In the US, the incidence of LN-associated ESRD increased 5 times approximately from 1982 to 2004 (Maroz and Segal, 2013). A need for careful monitoring of severe LN patients for progression to ESRD is recommended, including continuous immunosuppressive medication, regular follow-up, histopathologic 
examination, assessment of renal indices and treatment response of LN during maintenance (Hahn et al., 2012).

As a validation of our model, considering the most costeffective strategy (S3: CYC $\rightarrow$ AZA) and most effective strategy (S6: MMF $\rightarrow$ MMF), the risk of developing ESRD were 4.0 and $2.8 \%$ respectively by 6 years, consistent with a meta-analysis analyzing studies with follow-up from 3 to 6 years, in which the pooled risk of developing ESRD were 30 and 17 per 1,000 during maintenance therapy using AZA and MMF respectively (Tunnicliffe et al., 2018). The estimated risks of 10 -years all-cause death were 11.8 and $10.7 \%$ under treatment with S3 (CYC $\rightarrow$ AZA) and S6 (MMF $\rightarrow \mathrm{MMF),} \mathrm{similar} \mathrm{to}$ another epidemiological study showing that the patient survival in Asia (Hong Kong, Iran, and Japan) reached 92\% with the effect of immunosuppressive therapies over the same time span (Yap and Chan, 2015).

Our study has several limitations. First, some parameters were not available from China. We used available data from other countries which were most relevant. Heath-related quality of life in $\mathrm{LN}$ patients was estimated from several other countries. Second, the dosage of CYC in maintenance therapy was not obtained from official guidelines as it is no longer recommended as first-line therapy (Hahn et al., 2012; Chinese Guidelines for Diagnostic and Treatment of Lupus Nephritis Writing Group, 2019). We assumed the decrease to half of the dosage in the maintenance phase was reflected in the drug price. Sensitivity analysis also showed that the cost of CYC had limited impact on the results. Third, some losses were difficult to measure in terms of exact costs, such as ovarian failure due to CYC where there is no effective way of prevention and treatment $(\mathrm{McDermott}$ and Powell, 1996). We also did not consider withdrawal of therapy due to more severe but rare AEs or other reasons. For example, monitoring of peripheral $\mathrm{T}$ lymphocytes are recommended when patients receive immunosuppression therapy (Houssiau et al., 2010). Dose reduction or even withdrawal of MMF should be considered if lymphocytes continue to decline, or CD4 + T cells are less than $200 / \mu \mathrm{L}$ (Chinese Guidelines for Diagnostic and Treatment of Lupus Nephritis Writing Group, 2019). Lastly, though we have restricted our analysis to class III, IV, and III/ $\mathrm{IV}+\mathrm{V}$ LN patients and considered combination of drug options at the induction and maintenance therapy in each of which patients were more homogeneous in terms of disease severity, we could not rule out residual confounding by indication. It is also uncertain whether IS drug failure in the induction therapy would modify the efficacy of another IS drugs in the following induction or maintenance therapy, and we assumed efficacy of each therapy was independent.

\section{REFERENCES}

Adler, M., Chambers, S., Edwards, C., Neild, G., and Isenberg, D. (2006). An Assessment of Renal Failure in an SLE Cohort with Special Reference to Ethnicity, over a 25-year Period. Rheumatology (Oxford) 45 (9), 1144-1147. doi:10.1093/rheumatology/kel039

Almaani, S., Meara, A., and Rovin, B. H. (2017). Update on Lupus Nephritis. Clin. J. Am. Soc. Nephrol. 12 (5), 825-835. doi:10.2215/CJN.05780616
In conclusion, our study demonstrated that for both a 3-years and lifetime horizon, the most cost-effective strategy for treating severe LN patients in China was CYC induction therapy, followed by AZA maintenance therapy at the three times GDP per capita WTP threshold. The strategy of using MMF in both induction and maintenance became cost-effective under the one times GDP per capita WTP threshold for a lifetime horizon, with clinical benefits of achieving the lowest ESRD and mortality among strategies considered. Monitoring of patients during maintenance for progression to ESRD is recommended.

\section{DATA AVAILABILITY STATEMENT}

The original contributions presented in the study are included in the article/Supplementary Material, further inquiries can be directed to the corresponding authors.

\section{AUTHOR CONTRIBUTIONS}

ZD: Study design, data acquisition, data analysis and manuscript drafting; XZ: Data acquisition, data analysis and data interpretation; IW: Data interpretation and manuscript revision; EL: Study design, data interpretation, manuscript drafting, revision and approval; ZL: Study design, manuscript revision and approval. All authors critical approved the final manuscript.

\section{FUNDING}

This work was supported by the "Ten and Five" Project of The Third Affiliated Hospital of Sun Yat-sen University (Grant No. SW201901).

\section{ACKNOWLEDGMENTS}

We thank all doctors and technicians for supporting the study.

\section{SUPPLEMENTARY MATERIAL}

The Supplementary Material for this article can be found online at: https://www.frontiersin.org/articles/10.3389/fphar.2021.678301/ full\#supplementary-material

Carls, G., Li, T., Panopalis, P., Wang, S., Mell, A. G., Gibson, T. B., et al. (2009). Direct and Indirect Costs to Employers of Patients with Systemic Lupus Erythematosus with and without Nephritis. J. Occup. Environ. Med. 51 (1), 66-79. doi:10.1097/JOM.0b013e31818a405a

Chen, Q., Ayer, T., and Chhatwal, J. (2016). Sensitivity Analysis in Sequential Decision Models. Med. Decis. Making 37 (2), 243-252. doi:10.1177/0272989X16670605

Chhatwal, J., Jayasuriya, S., and Elbasha, E. H. (2016). Changing Cycle Lengths in State-Transition Models: Challenges and Solutions. Med. Decis. Making 36 (8), 952-964. doi:10.1177/0272989X16656165 
Chinese Guidelines for Diagnostic and Treatment of Lupus Nephritis Writing Group (2019). Chinese Guidelines for Diagnostic and Treatment of Lupus Nephritis. Natl. Med. J. China.

Contreras, G., Pardo, V., Leclercq, B., Lenz, O., Tozman, E., O’Nan, P., et al. (2004). Sequential Therapies for Proliferative Lupus Nephritis. N. Engl. J. Med. 350 (10), 971-980. doi:10.1056/NEJMoa031855

Croca, S. C., Rodrigues, T., and Isenberg, D. A. (2011). Assessment of a Lupus Nephritis Cohort over a 30-year Period. Rheumatology (Oxford) 50 (8), 1424-1430. doi:10.1093/rheumatology/ker101

Dooley, M. A., Jayne, D., Ginzler, E. M., Isenberg, D., Olsen, N. J., Wofsy, D., et al. (2011). Mycophenolate versus Azathioprine as Maintenance Therapy for Lupus Nephritis. N. Engl. J. Med. 365 (20), 1886-1895. doi:10.1056/NEJMoa1014460

Fanouriakis, A., Kostopoulou, M., Alunno, A., Aringer, M., Bajema, I., Boletis, J. N., et al. (2019). 2019 Update of the EULAR Recommendations for the Management of Systemic Lupus Erythematosus. Ann. Rheum. Dis. 78 (6), 736-745. doi:10.1136/annrheumdis-2019-215089

Hahn, B. H., McMahon, M. A., Wilkinson, A., Wallace, W. D., Daikh, D. I., Fitzgerald, J. D., et al. (2012). American College of Rheumatology Guidelines for Screening, Treatment, and Management of Lupus Nephritis. Arthritis Care Res. 64, 797-808. doi:10.1002/acr.21664

Houssiau, F. A., D'Cruz, D., Sangle, S., Remy, P., Vasconcelos, C., Petrovic, R., et al. (2010). Azathioprine versus Mycophenolate Mofetil for Long-Term Immunosuppression in Lupus Nephritis: Results from the MAINTAIN Nephritis Trial. Ann. Rheum. Dis. 69 (12), 2083-2089. doi:10.1136/ ard.2010.131995

Jo, C. (2014). Cost-of-illness Studies: Concepts, Scopes, and Methods. Clin. Mol. Hepatol. 20 (4), 327-337. doi:10.3350/cmh.2014.20.4.327

Jones, R. B., Hiemstra, T. F., Ballarin, J., Blockmans, D. E., Brogan, P., Bruchfeld, A., et al. (2019). Mycophenolate Mofetil versus Cyclophosphamide for Remission Induction in ANCA-Associated Vasculitis: a Randomised, Non-inferiority Trial. Ann. Rheum. Dis. 78 (3), 399-405. doi:10.1136/annrheumdis-2018214245

Korbet, S. M., Lewis, E. J., Schwartz, M. M., Reichlin, M., Evans, J., and Rohde, R. D. (2000). Factors Predictive of Outcome in Severe Lupus Nephritis. Lupus Nephritis Collaborative Study Group. Am. J. Kidney Dis. 35 (5), 904-914. doi:10.1016/s0272-6386(00)70262-9

Liem, Y. S., Bosch, J. L., and Hunink, M. G. (2008). Preference-based Quality of Life of Patients on Renal Replacement Therapy: a Systematic Review and MetaAnalysis. Value Health 11 (4), 733-741. doi:10.1111/j.1524-4733.2007.00308.x

Liu, L. L., Jiang, Y., Wang, L. N., Yao, L., and Li, Z. L. (2012). Efficacy and Safety of Mycophenolate Mofetil versus Cyclophosphamide for Induction Therapy of Lupus Nephritis: a Meta-Analysis of Randomized Controlled Trials. Drugs 72 (11), 1521-1533. doi:10.2165/11635030-000000000-00000

Maroz, N., and Segal, M. S. (2013). Lupus Nephritis and End-Stage Kidney Disease. Am. J. Med. Sci. 346 (4), 319-323. doi:10.1097/MAJ.0b013e31827f4ee3

Marseille, E., Larson, B., Kazi, D. S., Kahn, J. G., and Rosen, S. (2015). Thresholds for the Cost-Effectiveness of Interventions: Alternative Approaches. Bull. World Health Organ. 93 (2), 118-124. doi:10.2471/BLT.14.138206

McDermott, E. M., and Powell, R. J. (1996). Incidence of Ovarian Failure in Systemic Lupus Erythematosus after Treatment with Pulse Cyclophosphamide. Ann. Rheum. Dis. 55 (4), 224-229. doi:10.1136/ard.55.4.224

Mohara, A., Pérez Velasco, R., Praditsitthikorn, N., Avihingsanon, Y., and Teerawattananon, Y. (2014). A Cost-Utility Analysis of Alternative Drug Regimens for Newly Diagnosed Severe Lupus Nephritis Patients in Thailand. Rheumatology (Oxford) 53 (1), 138-144. doi:10.1093/ rheumatology/ket304

Mok, C. C., Kwok, R. C., and Yip, P. S. (2013). Effect of Renal Disease on the Standardized Mortality Ratio and Life Expectancy of Patients with Systemic Lupus Erythematosus. Arthritis Rheum. 65 (8), 2154-2160. doi:10.1002/ art.38006

Moroni, G., Raffiotta, F., Trezzi, B., Giglio, E., Mezzina, N., Del Papa, N., et al. (2014). Rituximab vs Mycophenolate and vs Cyclophosphamide Pulses for Induction Therapy of Active Lupus Nephritis: a Clinical Observational Study. Rheumatology (Oxford) 53 (9), 1570-1577. doi:10.1093/rheumatology/ket462

National Bureau of Statistics of China (2019). Annual Data. Available at: http:// data.stats.gov.cn/.

Nee, R., Rivera, I., Little, D. J., Yuan, C. M., and Abbott, K. C. (2015). Cost-utility Analysis of Mycophenolate Mofetil versus Azathioprine Based Regimens for
Maintenance Therapy of Proliferative Lupus Nephritis. Int. J. Nephrol. 2015, 917567-917613. doi:10.1155/2015/917567

Nossent, H. C., and Koldingsnes, W. (2000). Long-term Efficacy of Azathioprine Treatment for Proliferative Lupus Nephritis. Rheumatology (Oxford) 39, 969-974. doi:10.1093/rheumatology/39.9.969

Parikh, S. V., Almaani, S., Brodsky, S., and Rovin, B. H. (2020). Update on Lupus Nephritis: Core Curriculum 2020. Am. J. Kidney Dis. 76 (2), 265-281. doi:10.1053/j.ajkd.2019.10.017

Pons-Estel, B. A., Bonfa, E., Soriano, E. R., Cardiel, M. H., Izcovich, A., Popoff, F., et al. (2018). First Latin American clinical practice guidelines for the treatment of systemic lupus erythematosus: Latin American Group for the Study of Lupus (GLADEL, Grupo Latino Americano de Estudio del Lupus)-Pan-American League of Associations of Rheumatology (PANLAR). Ann. Rheum. Dis. 77 (11), 1549-1557. doi:10.1136/annrheumdis-2018-213512

Swai, J., Zhao, X., Noube, J. R., and Ming, G. (2020). Systematic Review and MetaAnalysis of Clinical Outcomes Comparison between Different Initial Dialysis Modalities in End-Stage Renal Disease Patients Due to Lupus Nephritis Prior to Renal Transplantation. BMC Nephrol. 21, 156. doi:10.1186/s12882-02001811-y

The Third Affiliated Hospital of Sun Yat-sen University (2019). Hospital Information System. Available at: https://www.zssy.com.cn.

Tsai, W. T., Chang, H. C., Wang, C. T., Chiang, B. L., and Lin, Y. T. (2019). Longterm Outcomes in Lupus Patients Receiving Different Renal Replacement Therapy. J. Microbiol. Immunol. Infect. 52 (4), 648-653. doi:10.1016/ j.jmii.2018.12.010

Tunnicliffe, D. J., Palmer, S. C., Henderson, L., Masson, P., Craig, J. C., Tong, A., et al. (2018). Immunosuppressive Treatment for Proliferative Lupus Nephritis. Cochrane Database Syst. Rev. 6 (6), CD002922. doi:10.1002/ 14651858.CD002922.pub4

Wang, F., Yang, C., Long, J., Zhao, X., Tang, W., Zhang, D., et al. (2019). Executive Summary for the 2015 Annual Data Report of the China Kidney Disease Network (CK-NET). Kidney Int. 95 (3), 501-505. doi:10.1016/ j.kint.2018.11.011

Wang, H., Ren, Y. L., Chang, J., Gu, L., and Sun, L. Y. (2018). A Systematic Review and Meta-Analysis of Prevalence of Biopsy-Proven Lupus Nephritis. Arch. Rheumatol. 33 (1), 17-25. doi:10.5606/ArchRheumatol.2017.6127

Wang, J. J., Lu, Y. E., Shan-Lian, H. U., Hao, C. P., and Han, F. (2006). Disease burden on Dialysis Therapy. Chin. Health Resour.

Whitehead, S. J., and Ali, S. (2010). Health Outcomes in Economic Evaluation: the QALY and Utilities. Br. Med. Bull. 96 (1), 5-21. doi:10.1093/bmb/ldq033

Wilson, E. C., Jayne, D. R., Dellow, E., and Fordham, R. J. (2007). The CostEffectiveness of Mycophenolate Mofetil as Firstline Therapy in Active Lupus Nephritis. Rheumatology (Oxford) 46 (7), 1096-1101. doi:10.1093/ rheumatology/kem054

Wu, M. J., Lo, Y. C., Lan, J. L., Yu, T. M., Shu, K. H., Chen, D. Y., et al. (2014). Outcome of Lupus Nephritis after Entering into End-Stage Renal Disease and Comparison between Different Treatment Modalities: A Nationwide Population-Based Cohort Study in Taiwan. Transpl. Proc 46 (2), 339-341. doi:10.1016/j.transproceed.2013.11.080

Xiaoming, P., Heli, X., Chenguang, D., Hua, L., Guozhen, C., and Mao, T. (2012). Cost of Two Different Therapies for End-Stage Renal Disease in Northwest China. J. Med. Colleges PLA(China) 27 (2), 80-86.

Yap, D. Y., and Chan, T. M. (2015). Lupus Nephritis in Asia: Clinical Features and Management. Kidney Dis. (Basel) 1 (2), 100-109. doi:10.1159/000430458

Yap, D. Y., Tang, C. S., Ma, M. K., Lam, M. F., and Chan, T. M. (2012). Survival Analysis and Causes of Mortality in Patients with Lupus Nephritis. Nephrol. Dial. Transpl. 27 (8), 3248-3254. doi:10.1093/ndt/gfs073

Yikui, S., Ping, Z., Linge, H., Xinyue, Y., and Hongxiu, L. (2015). Effect of Maintenance Hemodialysis on Prognosis and Survival of Patients with EndStage Renal Disease. Shanxi Med. J.

Zhang, L., Lu, G. H., Ye, S., Wu, B., Shen, Y., and Li, T. (2017). Treatment Adherence and Disease burden of Individuals with Rheumatic Diseases Admitted as Outpatients to a Large Rheumatology center in Shanghai, China. Patient Prefer Adherence 11, 1591-1601. doi:10.2147/PPA.S144624

Zhang, L., and Zuo, L. (2016). Current burden of End-Stage Kidney Disease and its Future Trend in China. Clin. Nephrol. 86 (S1), 27-28. doi:10.5414/CNP86S104

Zhang, X. W., Li, C., Ma, X. X., Zhao, J. X., An, Y., Liu, S., et al. (2014). Shortinterval Lower-Dose Intravenous Cyclophosphamide as Induction and 
Maintenance Therapy for Lupus Nephritis: a Prospective Observational Study. Clin. Rheumatol. 33 (7), 939-945. doi:10.1007/s10067-014-2590-6

Conflict of Interest: The authors declare that the research was conducted in the absence of any commercial or financial relationships that could be construed as a potential conflict of interest.

Publisher's Note: All claims expressed in this article are solely those of the authors and do not necessarily represent those of their affiliated organizations, or those of the publisher, the editors and the reviewers. Any product that may be evaluated in this article, or claim that may be made by its manufacturer, is not guaranteed or endorsed by the publisher.

Copyright (c) 2021 Dai, Zhang, Wong, Lau and Lin. This is an open-access article distributed under the terms of the Creative Commons Attribution License (CC BY). The use, distribution or reproduction in other forums is permitted, provided the original author(s) and the copyright owner(s) are credited and that the original publication in this journal is cited, in accordance with accepted academic practice. No use, distribution or reproduction is permitted which does not comply with these terms. 


\section{GLOSSARY}

LN Lupus nephritis

SLE Systemic lupus erythematosus

ESRD End-stage renal disease

CMA Chinese Medical Association

ACR American College of Rheumatology

EULAR European League Against Rheumatism

HCQ Hydroxychloroquine

GC Glucocorticoids

IS Immunosuppressive

US United States

USD (\$) United States Dollar

CYC Cyclophosphamide

AZA Azathioprine

MMF Mycophenolate mofetil

RTX Rituximab
AE Adverse event

QALY Quality-adjusted life year

ICER Incremental cost-effectiveness ratio

HD Hemodialysis

PD Peritoneal dialysis

CNY (¥) Chinese Yuan

GDP Gross domestic product

WTP Willingness-to-pay

DSA Deterministic sensitivity analysis

PSA Probabilistic sensitivity analysis

S1 Strategy 1

S2 Strategy 2

S3 Strategy 3

S4 Strategy 4

S5 Strategy 5

S6 Strategy 6 